\title{
MANOBRAS EVASIVAS SUJEITA À NÃO ESFERICIDADE DA TERRA E A CONDIÇÕES PARA QUASE-RENDEZVOUS
}

\author{
Thamis Cortes Freire de Carvalho; Antônio Delson C. de Jesus ${ }^{2}$
}

1. Bolsista PIBITI-CNPq,Graduando em Física, Universidade Estadual de Feira de Santana, email: tham_mia@hotmail.com

2. Orientador, Departamento de Física, Universidade Estadual de Feira de Santana,email: a1d1j1@gmail.com

\section{INTRODUÇÃO}

\section{PALAVRAS-CHAVE:}

Manobras evasivas são utilizadas nas missões espaciais pelo sistema de propulsão dos veículos operacionais frente à colisão com detritos espaciais. Além das manobras evasivas outra categoria de manobras tem um papel importante nas missões espaciais. Essas manobras são conhecidas como manobras de Rendezvous. Elas servem para realizar encontros de veículos com veículos ou encontros de veículos com detritos espaciais para alcançar objetivos previstos diversos das missões como coletas de materiais, visitas de sondas a asteroides, etc. Contudo, devido à dinâmica do movimento relativo real tais manobras não são realizadas com perfeição, gerando em alguns casos pequenas colisões. Este tipo de fenômeno denominamos manobras de "QuaseRendezvous", que são manobras de encontro que são realizadas a velocidades muito pequenas, contudo, não nulas. Um estudo mais detalhado da resistência dos veículos espaciais a estes pequenos impactos carece de ser realizado em função, inclusive, das densidades dos materiais que formam as estruturas dos corpos colisionais, visando melhorias para as missões.

A não esfericidade do potencial gravitacional terrestre também pode ser um fator a ser considerado no ambiente de missões espaciais. Ela indica que a força da gravidade em um corpo em órbita não é direcionada perfeitamente para o centro do Terra. Enquanto o campo gravitacional de um planeta perfeitamente esférico depende apenas da distância do seu centro, o achatamento causa variações seculares em elementos orbitais dos corpos envolvidos. Esse parâmetro adimensional é conhecido como $\mathrm{J}_{2}$ o seu valor para o campo gravitacional terrestre é:

$$
I_{2}=0.00108263
$$

Em coordenadas cartesianas, o efeito do achatamento da Terra é dado por:

$$
\begin{gathered}
I_{2(r)}=\left[-\frac{3}{2}\left(J_{2} \mu R_{\varepsilon}^{2} / r^{4}\right)-\frac{9}{2}\left(J_{2} \mu R_{\varepsilon}^{2} / r^{4}\right) \operatorname{sen}^{2}(i) \operatorname{sen}^{2}(\theta)\right] \hat{\imath}+\left[\frac{6}{2}\left(J_{2} \mu R_{\varepsilon}^{2} / r^{4}\right) \operatorname{sen}^{2}(i) \cos (\theta)\right] \hat{\jmath} \\
+\left[\frac{6}{2}\left(J_{2} \mu R_{\varepsilon}^{2} / r^{4}\right) \operatorname{sen}(i) \operatorname{sen}(\theta)\right] \hat{k}
\end{gathered}
$$

onde i é a inclinação da órbita em relação ao Equador e $\theta$ é a anomalia verdadeira.

\section{MATERIAL E MÉTODOS OU METODOLOGIA}


A metodologia aplicada a este estudo é a mais simples possível. Trata-se da dedução das equações da dinâmica relativa entre os corpos envolvidos (DE e veículo espacial), considerando as forças gravitacional e a não-esfericidade da Terra. Logo após, a simulação numérica da dinâmica, com a obtenção da estatística das densidades dos materiais que formam os corpos colisionais, faz-se os gráficos e tabelas catálogo que indicam os limites para as manobras de Quase-Rendezvous. No final, a caracterização dos resultados em gráficos, tabelas, interpretações, publicação em periódicos e congressos científicos.

\section{RESULTADOS E/OU DISCUSSÃO}

Após as investigações, foram levantados catálogos com as densidades dos materiais que formam os detritos e os satélites como também as dimensões dos mesmos. O gráfico da Figura 1, a seguir, nos mostra como o tamanho dos objetos interfere no aumento das possibilidades de Quase-Rendezvous.
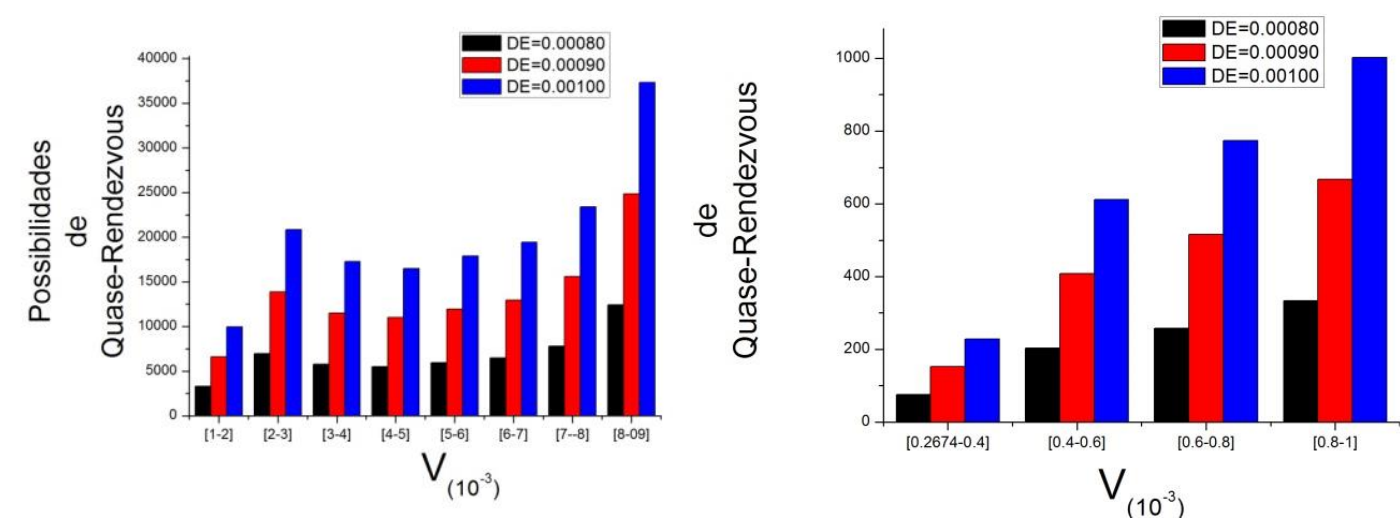

FIGURA 1- Faixas de pequenas velocidade vs. Possibilidades de Quase-Rendezvous para um satélite de raio 0,010Km e os DE's

Pretendendo estudar o efeito dessas colisões com velocidades baixas chegou-se a Equação (2) que permite determinar dissipação de energia em uma dinâmica colisional (P1, Vs - momentum linear e volume do satélite, respectivamente; P2, Vde - momentum linear e volume do detrito, respectivamente):

$$
\Delta E=\frac{\left(P_{1 \text { depois }}{ }^{2}-P_{1 \text { antes }}{ }^{2}\right)}{2 \rho_{s} V_{S}}+\frac{\left(P_{2 \text { depois }}{ }^{2}-P_{2 \text { antes }}{ }^{2}\right)}{2 \rho_{\text {de }} V_{d e}}
$$

A solução das equações do movimento, considerando a não-esfericidade da Terra são, em Coordenadas Cartesianas:

$$
\begin{aligned}
X_{(t)}=\left(x_{o}+\right. & \left.\left(\frac{C_{x}+C_{y}}{6 \omega^{2}}\right)+\left(\frac{C_{x}}{2 \omega^{2}}\right)-\left(\frac{2 C}{\omega}\right)-\left(\frac{H}{\omega^{2}}\right)\right) \cos (\omega t)+\left(\frac{\dot{x}}{\omega}\right) \sin (\omega t) \\
& -\left(\frac{C_{x}+C_{y}}{6 \omega^{2}}\right) \cos (2 \omega t)+\left(\left(\frac{C_{x}}{2 \omega^{2}}\right)+\left(\frac{2 C}{\omega}\right)+\left(\frac{H}{\omega^{2}}\right)\right)
\end{aligned}
$$




$$
\begin{aligned}
& Y_{(t)}=-2(-\left.3 x_{\circ}+\left(\frac{C_{x}+C_{y}}{6 \omega^{2}}\right)+\left(\frac{C_{x}+C_{y}}{2 \omega^{2}}\right)-\left(\frac{H}{\omega^{2}}\right)-\left(\frac{2 \dot{y}_{o}}{\omega}\right)\right) \sin (\omega t) \\
&-\left(\frac{2 \dot{x}_{o}}{\omega}\right)(1-\cos (\omega t))+\left(\frac{C_{x}+C_{y}}{6 \omega^{2}}+\left(\frac{C_{y}}{8 \omega^{2}}\right)\right) \sin (2 \omega t) \\
&+\left(-3 \dot{y}_{\circ}-6 \omega x_{\circ}-\left(\frac{2 H}{\omega}\right)+\left(\frac{C_{x}}{\omega}\right)+\left(\frac{C_{y}}{\omega}\right)-\left(\frac{C_{y}}{4 \omega}\right)\right) t \\
& Z_{(t)}=\left(z_{o}+\left(\frac{C_{z}}{2 \omega}\right) t\right) \cos (\omega t)+\left(\frac{z_{o}}{\omega}-\frac{C_{z}}{2 \omega^{2}}\right) \sin (\omega t)
\end{aligned}
$$

A Figura 2 mostra um histograma contendo condições iniciais para a colisão quando a inclinação da orbita em relação ao equador é igual a zero.

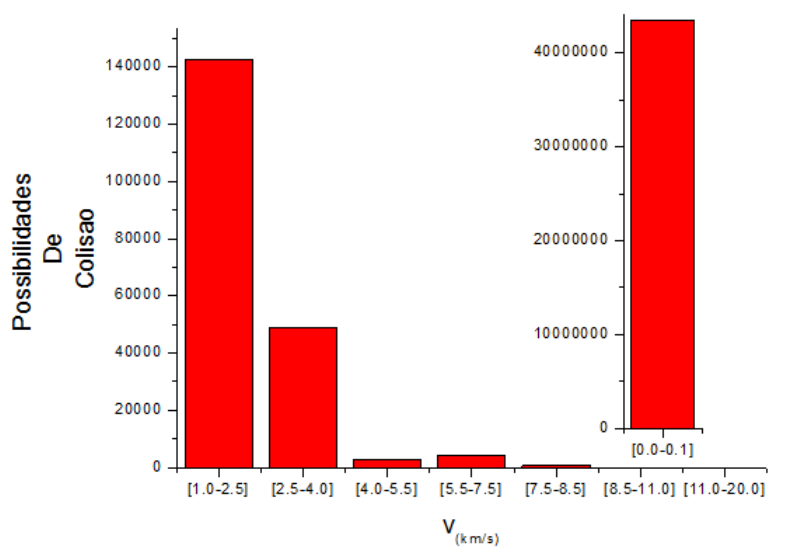

Figura 2 - Possibilidade de Colisão vs. Velocidades iniciais relativas para o caso $i=0^{\circ}$.

A solução das equações do movimento, considerando a não-esfericidade da Terra e a força de propulsão é (em Coordenadas Cartesianas):

$$
\begin{aligned}
& Z_{(t)}=\left(z_{o}-(\right.\left.\left.\frac{C_{z}}{2 \omega}\right)-v_{z} \sum_{n=1}^{\infty} \frac{k_{n}}{(\gamma n)^{2}+(\omega)^{2}}\right) \cos (\omega t) \\
&+\left(\frac{\dot{z}_{o}}{\omega}+\frac{v_{z}}{\omega} \ln \left(\frac{\chi+1}{\chi}\right)-v_{z} \sum_{n=1}^{\infty} \frac{\omega k_{n}}{(\gamma n)^{2}+(\omega)^{2}}-\left(\frac{C_{z}}{2 \omega^{2}}\right)\right) \sin (\omega t) \\
&+v_{z} \sum_{n=1}^{\infty} \frac{(\gamma n) k_{n}}{(\gamma n)^{2}+(\omega)^{2}} e^{-\gamma n t}+\frac{C_{z}}{2 \omega} \cos (\omega t) t \\
& X_{(t)=} A \cos (\omega t)+B \sin (\omega t)+\sum_{n=1}^{\infty} \frac{k_{n}}{\left((\omega)^{2}+(\gamma n)^{2}\right)}\left[v_{x} \gamma n-2 v_{y} \omega\right] e^{-\gamma n t}+\frac{2 v_{y}}{\omega} \ln \left(\frac{X+1}{\chi}\right) \\
& \quad+\left(\frac{H}{\omega^{2}}-\frac{C_{y}}{2 \omega^{2}}-\frac{C_{x}}{2 \omega^{2}}+4 x_{o}+\frac{2 \dot{y}_{o}}{\omega}\right)+\left(-\frac{C_{y}}{6 \omega^{2}}-\frac{C_{x}}{6 \omega^{2}}\right) \cos (2 \omega t) \\
& Y_{(t)=y_{o}-2 A} \operatorname{sen}(\omega t)-2 B(1-\cos (\omega t))-2 \omega \sum_{n=1}^{\infty} \frac{k_{n}\left[v_{x} \gamma n-2 v_{y} \omega\right]}{\left((\omega)^{2}+(\gamma n)^{2}\right)(\gamma n)}\left[1-e^{-\gamma n t}\right] \\
& \quad-v_{y} \sum_{n=1}^{\infty} \frac{k_{n}}{(\gamma n)}\left[1-e^{-\gamma n t}\right] \\
&+\left(-\frac{2 H}{\omega}+\frac{C_{y}}{\omega}+\frac{C_{x}}{\omega}-6 \omega x_{o}-3 \dot{y}_{o}-\frac{C_{y}}{4 \omega}-3 v_{y} \ln \left(\frac{\chi x+1}{\chi}\right)\right) t \\
&+\left(\frac{C_{y}}{6 \omega^{2}}+\frac{C_{x}}{6 \omega^{2}}+\frac{C_{y}}{8 \omega^{2}}\right) \operatorname{sen}(2 \omega t)
\end{aligned}
$$


$k_{n}=\frac{(-1)^{n+1}}{n \chi^{n}}$

Estas equações mostram a dinâmica de um satélite em ambiente de detritos espaciais, sujeito à força de propulsão e à não-esfericidade da Terra. Através dos parâmetros tecnológicos que caracterizam o sistema de propulsão, manobras evasivas poderão ser realizadas para evitar colisão do veículo com o detrito espacial.

\section{CONSIDERAÇÕES FINAIS}

Após os devidos estudos foi possível concluir que as dimensões dos detritos de os satélites influenciam de maneira direta na quantidade de colisão com detritos. Verificamos que detritos maiores aumentam as possibilidades de colisão (ou quaserendezvous) à medida em que as velocidades iniciais relativas aumentam. Deduzimos a equação da transferência de energia na colisão entre os corpos em função das densidades. As possibilidades de manobras de quase-rendezvous, considerando a não esfericidade da Terra, são abundantes para velocidades iniciais relativas pequenas. Este resultado pôde ser mostrado para órbitas no Equador $\left(\mathrm{i}=0^{\circ}\right)$. Ou seja, de fato, confirmamos pequenas colisões com baixas energias. Deduzimos também as equações do movimento e encontramos as soluções para a dinâmica, considerando a nãoesfericidade da Terra e a propulsão.

\section{REFERÊNCIAS}

Cordelli, A., Farinella, P. and Rossi, A. The influence of the fragmentation threshold on the long term evolution of the orbital debris environment (submitted to Elsevier Preprint, 1997).

Davis, D.R., Farinella, P., Paolicchi, P., Weidenschilling, S.J, and Binzel, R.P.: 1989, Asteroid collisional history: Effects on sizes and spins, in Asteroids II, eds. R.P.Binzel, T.Gehrels and M.S. Matthews(Univ. of Arizona Press), pp.805-826.

Davis, D. R., Farinella, P., Paolicchi, P., Weidenschiling, S. J. and Binzel, R. P. Asteroid collisional history: Effects on sizes and spins, in Asteroids II, edited by R. P. Binzel, T. Gérels, and M. S. Mattews, 805-826, University of Arizona Press, Tucson, 1989.

Davis, D. R., Ryan, E. V. and Farinella, P. Asteroid collisional evolution: Results from current scaling algoritms, Planet Space Sci., 42, 599-610, 1994.

Kessler, D. J., Cour-Palais, B.G. Collision frequency of artificial satellite: The creation of a debris belt, J. Geophys. Res., 83, 2637-2646, 1978.

Kessler, D. J., Landry, P.M., Cour-Palais, B.G., Taylor, R.E. Collision Avoidance in Space, IEEE Spectrum, June 80, 1980. 